\title{
Gray matter volume reduction in the chronic fatigue syndrome
}

\author{
Floris P. de Lange, ${ }^{\mathrm{a}, *}$ Joke S. Kalkman, ${ }^{\mathrm{b}}$ Gijs Bleijenberg, ${ }^{\mathrm{b}}$ Peter Hagoort, ${ }^{\mathrm{a}}$ \\ Jos W.M. van der Meer, ${ }^{\mathrm{c}}$ and Ivan Toni ${ }^{\mathrm{a}}$ \\ ${ }^{a}$ F.C. Donders Centre for Cognitive Neuroimaging, Radboud University Nijmegen, NL-6500 HB Nijmegen, The Netherlands \\ ${ }^{\mathrm{b}}$ Expert Center Chronic Fatigue, University Medical Center, Nijmegen, The Netherlands \\ ${ }^{\mathrm{c}}$ Department of Internal Medicine, University Medical Center, Nijmegen, The Netherlands
}

Received 28 September 2004; revised 11 January 2005; accepted 18 February 2005

Available online 7 April 2005

\begin{abstract}
The chronic fatigue syndrome (CFS) is a disabling disorder of unknown etiology. The symptomatology of CFS (central fatigue, impaired concentration, attention and memory) suggests that this disorder could be related to alterations at the level of the central nervous system. In this study, we have used an automated and unbiased morphometric technique to test whether CFS patients display structural cerebral abnormalities.

We mapped structural cerebral morphology and volume in two cohorts of CFS patients (in total 28 patients) and healthy controls (in total 28 controls) from high-resolution structural magnetic resonance images, using voxel-based morphometry. Additionally, we recorded physical activity levels to explore the relation between severity of CFS symptoms and cerebral abnormalities.

We observed significant reductions in global gray matter volume in both cohorts of CFS patients, as compared to matched control participants. Moreover, the decline in gray matter volume was linked to the reduction in physical activity, a core aspect of CFS.

These findings suggest that the central nervous system plays a key role in the pathophysiology of CFS and point to a new objective and quantitative tool for clinical diagnosis of this disabling disorder.

(C) 2005 Elsevier Inc. All rights reserved.
\end{abstract}

Keywords: Chronic fatigue syndrome; Voxel-based morphometry; Magnetic resonance imaging; Volumetric MRI

\section{Introduction}

The chronic fatigue syndrome (CFS) is characterized by a persistent or relapsing unexplained fatigue, of new or definite onset and lasting for at least 6 months (Fukuda et al., 1994). CFS is believed to affect roughly half a million people in the United States, and results in significant personal and economic morbidity.

\footnotetext{
* Corresponding author. Fax: +31243610652.

E-mail address: floris.delange@fcdonders.ru.nl (F.P. de Lange).

Available online on ScienceDirect (www.sciencedirect.com).
}

The etiology of CFS is still largely unknown, although infectious, immunological, neuroendocrine, sleep, and psychological mechanisms have been proposed to play a role (Afari and Buchwald, 2003). Several symptoms associated with CFS suggest a role for the central nervous system, notably the central fatigue, impairments in concentration, attention and memory, headache, and unrefreshing sleep. Accordingly, structural (Buchwald et al., 1992; Cope and David, 1996; Lange et al., 1999; Natelson et al., 1993; Schwartz et al., 1994a,b) and functional (de Lange et al., 2004; MacHale et al., 2000; Schmaling et al., 2003) neuroimaging studies have investigated possible cerebral correlates of CFS.

Studies of brain morphology in CFS have focused on (subcortical) white matter abnormalities, which manifest themselves as foci of bright intensity on T2-weighted MR scans. These studies provide conflicting evidence for cerebral abnormalities. Whereas some studies reported an increased number of subcortical white matter abnormalities associated with CFS (Buchwald et al., 1992; Lange et al., 1999; Natelson et al., 1993; Schwartz et al., 1994a), other studies observed equal numbers of white matter abnormalities in healthy volunteers and CFS patients (Cope et al., 1995; Cope and David, 1996). An important limitation of these morphological studies lies in the absence of an automated procedure. Assessment of cerebral atrophy using human observers and rating scales has a poor reproducibility among raters and low inter-rater reliability [on average 50\%] (Scheltens et al., 1997). Secondly, studies that observed MRI abnormalities could not link the abnormalities to physical CFS markers (Cope et al., 1995) which makes the functional significance and clinical utility of these findings uncertain.

In this study, we aimed to investigate brain morphology in CFS patients by relying on a fully automated observer-independent procedure, voxel-based morphometry (VBM) (Ashburner and Friston, 2000). VBM provides an unbiased and validated (Watkins et al., 2002) technique for measuring cerebral volume and tissue concentration from high-resolution structural magnetic resonance images, and it has been used to assess subtle morphological brain differences in different pathologies (Draganski et al., 2002; May et al., 1999; Yamasue et al., 2003). Furthermore, we collected data 
pertaining to the physical activity level in the CFS patients and a subgroup of controls using actigraphic assessment. Patients with CFS are markedly sedentary (van der Werf et al., 2000). Activity levels provide a quantitative measure of the severity of this aspect of CFS, so by acquiring information about the physical activity level, we could assess the relationship between severity of MRI abnormalities and physical inactivity within the CFS population. Having observed significant structural abnormalities in a first cohort of CFS patients, we tested the reliability of the observation by replicating the experiment in a second independent cohort of equal size.

\section{Methods}

\section{Participants}

We studied the experimental effect in two separate cohorts of patients and matched controls. Since CFS predominantly affects women (Afari and Buchwald, 2003), and given the differences in brain morphology and size between men and women (Gur et al., 1991), we restricted this study to female participants. The first cohort consisted of 13 female CFS patients (CFS: mean age 28.9 years, SD 6.1, range 19-37) and 15 age-, sex-, and education-matched healthy controls (HC: mean age 25.7 years, SD 6.5, range 19-42). The second cohort consisted of 15 older female CFS patients (mean age 43.9 years, SD 14.4, range 24-63) and 13 age-, sex-, and educationmatched healthy controls (mean age 43.4 years, SD 14.1, range 2268). All participants took part in the study after giving written informed consent according to institutional guidelines of the local ethics committee (CMO region Arnhem-Nijmegen, Netherlands) and in accordance with the Helsinki Declaration of 1975, as revised in 1983. Patients and controls were assessed by means of detailed history and investigation, and computer assessment of questionnaires. The physical activity level of 26 of the CFS patients and 14 of the $\mathrm{HC}$ was assessed by actometer measurements during 2 weeks preceding scanning. All patients conformed to the US Centers for Disease Control and Prevention (CDC) criteria for CFS (Fukuda et al., 1994). Participants who manifested psychiatric comorbodity (e.g., depression) were excluded from the study. None of the participants took any drugs that acted on the central nervous system. We collected information regarding self-reported disease severity and disease duration in all patients. Self-reported disease severity was assessed by the Checklist Individual Strength (CIS). The Checklist Individual Strength (CIS) is a 20 -item questionnaire that measures several aspects of fatigue (Dittner et al., 2004; Vercoulen et al., 1994). Self-reported disease duration reflects the number of years the CFS patient felt she had been affected by complaints of fatigue.

\section{Actigraphic measurements}

The actometer (Actilog V3.0) used is a motion-sensing device that can register and quantify human physical activity (Vercoulen et al., 1997). The actometer is small and light and has to be worn at the ankle. The small size makes it suitable for long-term continuous registrations. The actometer has a piezoelectric sensor that is sensitive in three directions. Accelerations of the sensor larger than a predefined threshold are considered as activity and are stored in an internal memory. Each second, the counter of the actometer is read and reset by the microcontroller. The integration counter is set at $5 \mathrm{~min}$ providing every $5 \mathrm{~min}$ an activity score that is stored in the internal memory of the actometer. At the end of the registration period, data are fed into an external computer. Participants wore the actometer day and night during at least a 14-day period. In order to retain 12 complete registration days, the first and last registration days were omitted for the analyses. A general physical activity score reflected the average physical activity level over the total 12-day time period and was expressed in the average number of accelerations per 5-min period.

\section{Imaging protocol}

High-resolution anatomical images (voxel size $=1 \mathrm{~mm}^{3}$ ) of the whole brain were acquired on a 1.5-T Siemens Sonata whole-body scanner (Erlangen, Germany) using a magnetization prepared rapid acquisition gradient echo sequence. Images were analyzed using VBM (Ashburner and Friston, 2000), a fully automated technique for computational analysis of differences in global and local gray and/or white matter volume. This method involved the following steps: (1) spatial normalization of all images to a standardized anatomical space by removing differences in overall size, position, and global shape; (2) extraction of gray and white matter from the normalized images; (3) correction for volume changes induced by normalization; and (4) analysis of differences in global and local gray and white matter volume across the whole brain. We applied an optimized method of VBM (Ashburner and Friston, 2000; Good et al., 2001) using the SPM2 package (www.fil.ion.ucl.ac.uk/spm) and special-purpose scripting tools (http://dbm.neuro.uni-jena.de/ vbm.html). The spatial normalization to the standard anatomical space was performed in a two-stage process. In the first step, we registered each image to the International Consortium for Brain Mapping template (Montreal Neurological Institute, Montreal, Canada), which approximates Talairach and Tournoux space (Talairach and Tournoux, 1988). We applied a 12-parameter affine transformation to correct for image size and position. Regional volumes were preserved while corrections for global differences in whole brain volume were made. The normalized images of all participants were averaged and smoothed with a Gaussian kernel of $8 \mathrm{~mm}$ full-width at half-maximum (FWHM) and then used as a new template with reduced scanner- and population-specific biases. In the second normalization step, we locally deformed each image of the whole group to the new template using a nonlinear spatial transformation. This accounts for the remaining shape differences between the images and the template and improves the overlap of corresponding anatomical structures. Finally, using a modified mixture model cluster analysis, normalized images were corrected for nonuniformities in signal intensity and partitioned into gray and white matter, cerebrospinal fluid, and background. To remove unconnected non-brain voxels (e.g., rims between brain surface and meninges), we applied a series of morphological erosions and dilations to the segmented images (Good et al., 2001). To correct for possible volume changes as a result of the nonlinear spatial normalization, all images were modulated by multiplying voxel values in the segmented images by the Jacobian determinants derived from the spatial normalization step (Good et al., 2001). The resulting modulated images were smoothed with a Gaussian kernel of $12 \mathrm{~mm}$ FWHM. Global volume was calculated from these modulated images. Head size was estimated by means of a semiautomated procedure. First, the original MR scans of each participant were aligned to a common stereotactic space defined by the plane passing through the anterior and posterior commis- 


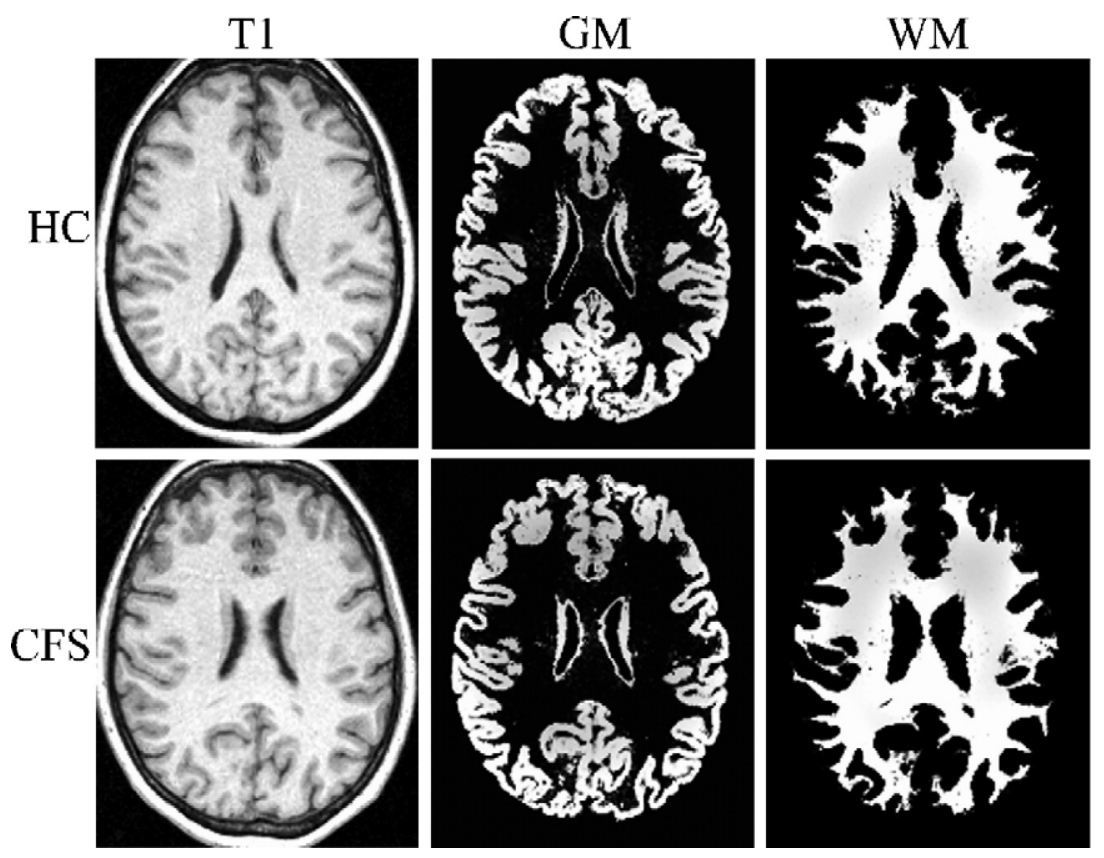

Fig. 1. Voxel-based morphometric analysis. Data are shown for one participant of the healthy control group (HC, upper row) and the patient group (CFS, lower row). We segmented high-resolution anatomical images (left column) into gray matter (GM, middle column), white matter (WM, right column), cerebrospinal fluid, and non-brain tissue (not shown).

sures (Talairach and Tournoux, 1988). Second, we manually defined the transverse and sagittal diameters of the skull along this plane. Head size was calculated as the product of these two diameters. Self-reported disease severity was taken from the US Centers for Disease Control and Prevention questionnaire.

\section{Statistical analysis}

Global differences in gray and white matter between groups were assessed with an analysis of covariance (ANCOVA) that considered age as a confounding covariate. Regional (i.e., voxelby-voxel) differences in gray matter between groups were assessed with $t$ tests using the general linear model, considering age and total gray matter volume as confounding covariates, and correcting the results for search volume by using family-wise error correction. Head size differences were assessed with an analysis of variance (ANOVA). The correlation between daily physical activity and gray matter volume was assessed by means of Pearson's correlation coefficients.

\section{Results}

Fig. 1 shows two examples of high-resolution anatomical images, and the resulting gray matter (GM) and white matter (WM) maps derived from the automated segmentation procedure as described in the Methods section.

Both cohorts of CFS patients showed significant reductions in gray matter volume (GM) compared to $\mathrm{HC}$ [cohort I: $F_{(1,25)}=6.2$; $P=0.019$; cohort II: $\left.F_{(1,25)}=8.0 ; P=0.009\right]$. When the two cohorts are put together, the GM reduction remains highly significant $\left[F_{(1,53)}=14.5 ; P<0.001\right]$ and amounts to a reduction in GM of approximately $8 \%$ [95\% Confidence Interval: 5-11\%] (Fig. 2A). In terms of age-related changes in GM, both CFS
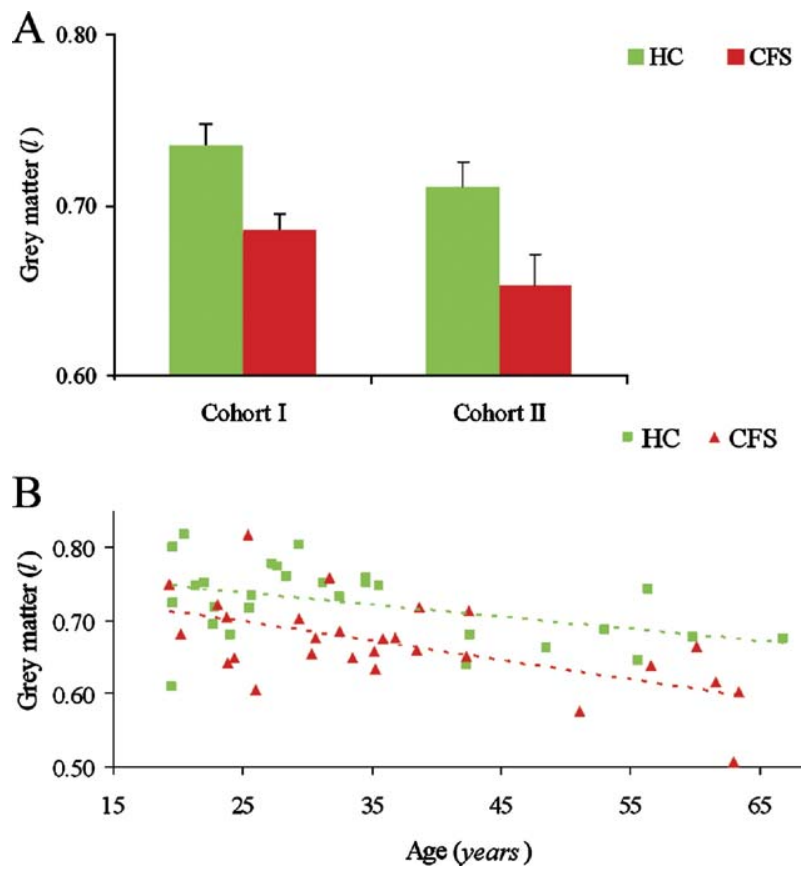

Fig. 2. Gray matter volume. (A) Gray matter volume (in liters; mean \pm SE) of two cohorts of patients suffering from the chronic fatigue syndrome (CFS, in red) and healthy control participants (HC, in green). In both cohorts, CFS patients have markedly reduced gray matter volumes (GM) than their healthy counterparts. The second cohort of CFS and HC participants is older than the first, giving rise to overall lower GM. (B) Scatterplot of gray matter volume as a function of age in the two CFS and $\mathrm{HC}$ cohorts. Both CFS patients and HC show a similar decline in GM as a function of age. 


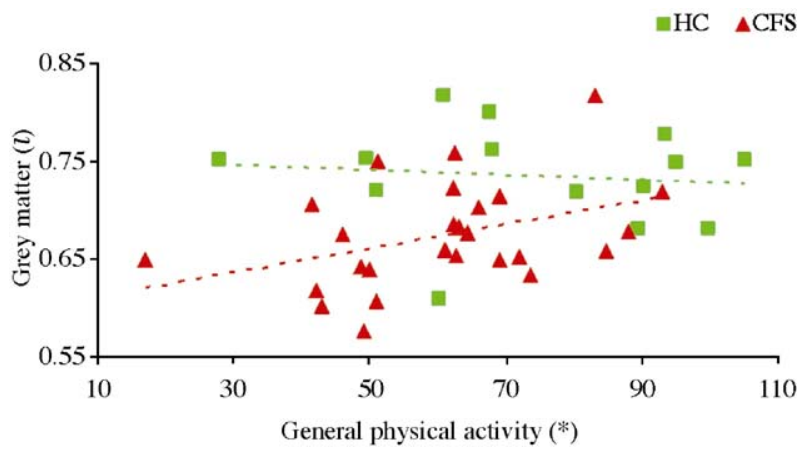

Fig. 3. Relation between physical activity and gray matter volume. There is a positive relationship between the amount of general physical activity and the gray matter volume within the CFS population (in red), but not in the healthy control group (in green). (*) General physical activity measured over a 2 -week period with a movement-sensing device, expressed in number of accelerations per 5-min period.

patients and $\mathrm{HC}$ show a similar decline in GM as a function of age $\left[F_{(1,53)}=19.5 ; P<0.001\right]$ (Fig. 2B). This decline amounts to a reduction of approximately $2.2 \mathrm{ml} /$ year, a decline that is in line with previous reports on age-related GM loss (Good et al., 2001). Across the wide age range of our sample (19-66 years), there was no indication of group $\times$ age interactions $\left[F_{(1,52)}=1.01 ; P=\right.$ $0.32]$, i.e., the GM reduction observed in the CFS groups was not due to a faster age-related decline.

We did not detect regionally specific difference in GM (over and above global GM differences, with a threshold of $P<0.05$ corrected for search volume). A further exploratory analysis of statistical trends $(P<0.2$ corrected for search volume) failed to reveal local foci of reduced GM. Taken together, these findings suggest that the observed GM reduction in the CFS patient groups is a global rather than a local phenomenon, although further studies with larger statistical power might be required to detect subtle focal structural alterations. White matter volume was not significantly different between groups $\left[F_{(1,53)}=2.58 ; P=0.114\right]$, even when taking both cohorts together, indicating that the difference between groups is specific to GM. Overall head size was not significantly different between $\mathrm{HC}$ and CFS patients $\left[F_{(1,53)}=2.57 ; P=0.115\right]$, indicating that the differences between groups measured are not a by-product of differences in head size.

Within the CFS population, there was a positive correlation between daily physical activity level and GM (Fig. 3; $r=0.39$, $P=0.026$ one-tailed). Conversely, there was no significant correlation between physical activity level and GM in the control group (Fig. $3 ; r=-0.10, P=0.37$ one-tailed). There was no significant correlation between age and physical activity in the CFS group $(r=-0.11, P=0.30$ one-tailed), which excludes that the positive correlation between GM and physical activity in CFS patients is a by-product of age-related effects. There were no significant correlations between GM and self-reported CFS duration ( $r=0.02, P=0.46$ one-tailed) or CFS severity as measured by the Checklist Individual Strength $(r=0.20, P=0.16$ one-tailed).

\section{Discussion}

By using an automated morphometric technique, we were able to assess group-related differences across the whole brain in an observer-independent manner. Two independent cohorts of CFS patients showed a marked decline in gray matter volume (GM), compared to matched healthy controls. Moreover, although GM was not related to self-reported disease duration or severity, there was a relation between the (objectively measured) level of physical activity and the GM reduction.

The results obtained with this approach corroborate and complement previous studies that observed cerebral abnormalities associated with CFS (Buchwald et al., 1992; Lange et al., 1999; Natelson et al., 1993; Schwartz et al., 1994a). Our findings appear to provide a reliable somatic marker of CFS, and suggest that the key to this disease might lay in the central nervous system. However, given the multidimensional nature of CFS (Afari and Buchwald, 2003), the relationship between the structural brain abnormalities in CFS and the etiology of this disorder may not be straightforward. It is conceivable that the reduced gray matter volume is a cause of chronic fatigue and the ensuing physical inactivity. Alternatively, it is also possible that the gray matter reduction is a consequence of the reduced physical activity inherent in CFS. In this perspective, recent reports have suggested direct links between physical exercise and neurogenesis (Pham et al., 2003; van Praag et al., 1999). However, the latter scenario is not immediately compatible with the fact that the GM decrease is not correlated with CFS duration. Future research is clearly warranted to assess whether the present results can be validated in bigger CFS populations and whether the changes we observe can be influenced by therapeutic intervention.

In conclusion, we found substantial and consistent reductions in GM volume in two independent cohorts of CFS patients. This GM reduction was associated to the decline in physical activity in the CFS patients. These findings suggest that the central nervous system plays a crucial role in the etiology of CFS. Furthermore, they provide a new objective and quantitative diagnostic marker of this disabling disorder.

\section{Acknowledgments}

This study was supported by the Netherlands ME foundation (ME Fonds, grant number W1101/16E) and by NWO (VIDI grant no. 452-03-339). The authors thank J. Ashburner for his support with methodological issues, R.C.G. Helmich, Karl Magnus Petersson, and S. van der Werf for helpful comments.

\section{References}

Afari, N., Buchwald, D., 2003. Chronic fatigue syndrome: a review. Am. J. Psychiatry 160, 221-236.

Ashburner, J., Friston, K.J., 2000. Voxel-based morphometry-the methods. NeuroImage 11, 805-821.

Buchwald, D., Cheney, P.R., Peterson, D.L., Henry, B., Wormsley, S.B., Geiger, A., Ablashi, D.V., Salahuddin, S.Z., Saxinger, C., Biddle, R., 1992. A chronic illness characterized by fatigue, neurologic and immunologic disorders, and active human herpesvirus type 6 infection. Ann. Intern. Med. 116, 103-113.

Cope, H., David, A.S., 1996. Neuroimaging in chronic fatigue syndrome. J. Neurol., Neurosurg. Psychiatry 60, 471-473.

Cope, H., Pernet, A., Kendall, B., David, A., 1995. Cognitive functioning and magnetic resonance imaging in chronic fatigue. Br. J. Psychiatry $167,86-94$. 
de Lange, F.P., Kalkman, J.S., Bleijenberg, G., Hagoort, P., van der Werf, S.P., van der Meer, J.W., Toni, I., 2004. Neural correlates of the chronic fatigue syndrome - an fMRI study. Brain 127, 1948-1957.

Dittner, A.J., Wessely, S.C., Brown, R.G., 2004. The assessment of fatigue: a practical guide for clinicians and researchers. J. Psychosom. Res. 56, $157-170$.

Draganski, B., Geisler, P., Hajak, G., Schuierer, G., Bogdahn, U., Winkler, J., May, A., 2002. Hypothalamic gray matter changes in narcoleptic patients. Nat. Med. 8, 1186-1188.

Fukuda, K., Straus, S.E., Hickie, I., Sharpe, M.C., Dobbins, J.G., Komaroff, A., 1994. The chronic fatigue syndrome: a comprehensive approach to its definition and study. International Chronic Fatigue Syndrome Study Group. Ann. Intern. Med. 121, 953-959.

Good, C.D., Johnsrude, I.S., Ashburner, J., Henson, R.N.A., Friston, K.J., Frackowiak, R.S.J., 2001. A voxel-based morphometric study of ageing in 465 normal adult human brains. NeuroImage 14, 21-36.

Gur, R.C., Mozley, P.D., Resnick, S.M., Gottlieb, G.L., Kohn, M., Zimmerman, R., Herman, G., Atlas, S., Grossman, R., Berretta, D., 1991. Gender differences in age effect on brain atrophy measured by magnetic resonance imaging. Proc. Natl. Acad. Sci. U. S. A. 88, 2845-2849.

Lange, G., DeLuca, J., Maldjian, J.A., Lee, H., Tiersky, L.A., Natelson, B.H., 1999. Brain MRI abnormalities exist in a subset of patients with chronic fatigue syndrome. J. Neurol. Sci. 171, 3-7.

MacHale, S.M., Lawrie, S.M., Cavanagh, J.T., Glabus, M.F., Murray, C.L., Goodwin, G.M., Ebmeier, K.P., 2000. Cerebral perfusion in chronic fatigue syndrome and depression. Br. J. Psychiatry 176, 550-556.

May, A., Ashburner, J., Buchel, C., McGonigle, D.J., Friston, K.J., Frackowiak, R.S., Goadsby, P.J., 1999. Correlation between structural and functional changes in brain in an idiopathic headache syndrome. Nat. Med. 5, 836-838.

Natelson, B.H., Cohen, J.M., Brassloff, I., Lee, H.J., 1993. A controlled study of brain magnetic resonance imaging in patients with the chronic fatigue syndrome. J. Neurol. Sci. 120, 213-217.

Pham, K., Nacher, J., Hof, P.R., McEwen, B.S., 2003. Repeated restraint stress suppresses neurogenesis and induces biphasic PSA-NCAM expression in the adult rat dentate gyrus. Eur. J. Neurosci. 17, 879-886.

Scheltens, P., Pasquier, F., Weerts, J.G., Barkhof, F., Leys, D., 1997. Qualitative assessment of cerebral atrophy on MRI: inter- and intraobserver reproducibility in dementia and normal aging. Eur. Neurol. 37, $95-99$.
Schmaling, K.B., Lewis, D.H., Fiedelak, J.I., Mahurin, R., Buchwald, D.S., 2003. Single-photon emission computerized tomography and neurocognitive function in patients with chronic fatigue syndrome. Psychosom. Med. 65, 129-136.

Schwartz, R.B., Garada, B.M., Komaroff, A.L., Tice, H.M., Gleit, M., Jolesz, F.A., Holman, B.L., 1994a. Detection of intracranial abnormalities in patients with chronic fatigue syndrome: comparison of MR imaging and SPECT. AJR Am. J. Roentgenol. 162, 935-941.

Schwartz, R.B., Komaroff, A.L., Garada, B.M., Gleit, M., Doolittle, T.H., Bates, D.W., Vasile, R.G., Holman, B.L., 1994b. SPECT imaging of the brain: comparison of findings in patients with chronic fatigue syndrome, AIDS dementia complex, and major unipolar depression. AJR Am. J. Roentgenol. 162, 943-951.

Talairach, J., Tournoux, P., 1988. Co-Planar Stereotaxic Atlas of the Human Brain: 3-Dimensional Proportional System: An Approach to Medical Cerebral Imaging. Thieme, Stuttgart.

van der Werf, S.P., Prins, J.B., Vercoulen, J.H., van der Meer, J.W., Bleijenberg, G., 2000. Identifying physical activity patterns in chronic fatigue syndrome using actigraphic assessment. J. Psychosom. Res. 49, $373-379$.

van Praag, H., Kempermann, G., Gage, F.H., 1999. Running increases cell proliferation and neurogenesis in the adult mouse dentate gyrus. Nat. Neurosci. 2, 266-270.

Vercoulen, J.H., Swanink, C.M., Fennis, J.F., Galama, J.M., van der Meer, J.W., Bleijenberg, G., 1994. Dimensional assessment of chronic fatigue syndrome. J. Psychosom. Res. 38, 383-392.

Vercoulen, J.H., Bazelmans, E., Swanink, C.M., Fennis, J.F., Galama, J.M., Jongen, P.J., Hommes, O., van der Meer, J.W., Bleijenberg, G., 1997. Physical activity in chronic fatigue syndrome: assessment and its role in fatigue. J. Psychiatr. Res. 31, 661-673.

Watkins, K.E., Vargha-Khadem, F., Ashburner, J., Passingham, R.E., Connelly, A., Friston, K.J., Frackowiak, R.S., Mishkin, M., Gadian, D.G., 2002. MRI analysis of an inherited speech and language disorder: structural brain abnormalities. Brain 125, 465-478.

Yamasue, H., Kasai, K., Iwanami, A., Ohtani, T., Yamada, H., Abe, O., Kuroki, N., Fukuda, R., Tochigi, M., Furukawa, S., Sadamatsu, M., Sasaki, T., Aoki, S., Ohtomo, K., Asukai, N., Kato, N., 2003. Voxelbased analysis of MRI reveals anterior cingulate gray-matter volume reduction in posttraumatic stress disorder due to terrorism. Proc. Natl. Acad. Sci. U. S. A. 100, 9039-9043. 